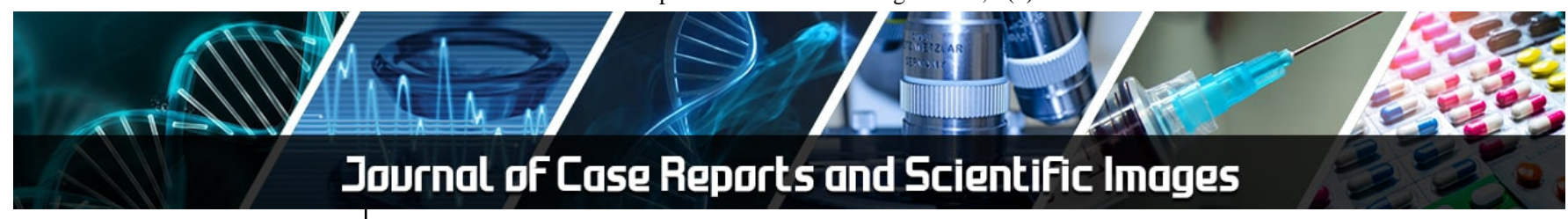

E-ISSN: 2708-0064

P-ISSN: 2708-0056

IJCRS 2021; 3(2): 01-04

http://www.allcasereports.com

Received: 20-08-2021

Accepted: 07-09-2021

Dr. Muni Bhavani Itha

Assistant Professor,

Department of Pathology,

Katuri Medical College and

Hospital, Guntur, Andhra

Pradesh, India

Dr. Yarlagadda Krishna

Bharathi

Professor, Department of

Pathology, Katuri Medical

College and Hospital, Guntur,

Andhra Pradesh, India

Dr. Satyanarayana

Veeragandham

Professor and Head,

Department of Pathology,

Katuri Medical College and

Hospital, Guntur, Andhra

Pradesh, India

\section{Coexisting upper tract urothelial carcinoma and renal tuberculosis: A rare presentation}

\author{
Dr. Muni Bhavani Itha, Dr. Yarlagadda Krishna Bharathi and Dr. \\ Satyanarayana Veeragandham
}

DOI: https://doi.org/10.22271/27080056.2021.v3.i2a.28

\begin{abstract}
Tuberculosis (TB) is a global public health problem and is a leading cause of death, especially in underdeveloped and developing countries. In the majority of instances, renal tuberculosis is caused by the secondary hematogenous transmission of mycobacterium from pulmonary lesions to the renal cortex either during the initial TB infection or reactivation/endogenous reinfection of an old tuberculous focus. Upper tract urothelial carcinoma is a relatively rare kind of genitourinary malignancy and the majority of them take their origin from the urothelial lining epithelium. The cooccurrence of both renal tuberculosis and upper tract urothelial carcinoma is a rare presentation, and several theories have been proposed to demonstrate the etiological relationship between these two conditions. Here we reported a case of co-existing renal tuberculosis and urothelial carcinoma in a 54year-old female with a remote history of treated pulmonary tuberculosis and also discussed the possible etiological root causes for their co-occurrence.
\end{abstract}

Keywords: tuberculosis, renal, transitional cell carcinoma

\section{Introduction}

It is quite uncommon for renal tuberculosis and urothelial carcinoma to occur in the same kidney. Only a few cases had previously been published in the literature, to our knowledge. Here we present, a case of a 54-year-old female patient who had both renal tuberculosis (renal TB) and upper tract urothelial carcinoma (UTUC) in the same kidney on the left side.

\section{Case Report}

A 54-year-old woman, reported with a two-month history of lower urinary tract symptoms such as increased urine frequency, urgency, and dysuria, as well as periodic episodes of fever accompanied by chills and rigors. She also reported a recent history of significant weight loss, appetite loss, as well as a history of dull painful discomfort in the left loin region with several episodes of painless hematuria of mild degree. She was not diabetic, but she was hypertensive and on medication. She had a distant history of treated pulmonary tuberculosis with no significant sequelae. Then the patient was advised for CECT urography/KUB.

CT scan revealed $3 \mathrm{X} 1.9 \mathrm{cms}$ heterogeneously enhancing soft tissue density in the ureter and also reported moderate hydroureteronephrosis [Fig 1] with calculus/urolith noted distal to the soft tissue density. On CTKUB, the possible differential diagnosis given for the soft tissue density in the ureter was 1) inflammatory granulation tissue 2) Neoplastic etiology - Ureter growth. Urine cytology revealed urine microscopy was negative for malignant cells. Ureteroscopy findings revealed polypoidal growth noted in the ureter and biopsy was taken from the representative site and sent for histopathological examination.

Histopathological examination of ureteric growth biopsy revealed papillary urothelial carcinoma of low malignant potential. (H.P No.171/2021).

Then the patient underwent laparoscopic left Nephroureterectomy and the resected specimen was sent to the histopathology section of our pathology department.

- Macroscopic examination of laparoscopic left Nephroureterectomy [H.P.NO 389/2021]: revealed kidney with perinephric fat altogether measuring $14 x 8 \times 6 \mathrm{cms}$ with attached ureter measuring $21 \mathrm{cms}$ in length. Cut section of kidney shows discretely scattered multiple greyish white nodules of size ranging from $1 \mathrm{~mm}$ to $7 \mathrm{~mm}$ in diameter. Also, the renal pelvis showed dilated pelvicalyceal system. Also noted, tiny papillary growth in the distal ureter $15 \mathrm{~cm}$ from the ureteropelvic junction and $6 \mathrm{cms}$ from the distal resected end of the ureter. [Fig:2-4]
Corresponding Author: Dr. Muni Bhavani Itha Assistant Professor, Department of Pathology, Katuri Medical College and Hospital, Guntur, Andhra Pradesh, India 
- Microscopic examination of laparoscopic left Nephroureterectomy [H.P.NO.389/2021]: revealed renal parenchyma showing multiple granulomas composed of epithelioid histiocytes, Langhans type of multinucleated giant cells and lymphoid aggregates, and focal granulomas associated with caseous necrosis. Photomicrograph of sections taken from ureter showed histological features of Non-Invasive Papillary Urothelial Carcinoma of Low Malignant Potential. After complete and detailed Histopathological examination revealed the diagnosis of coexisting Renal Tuberculosis and Papillary Urothelial Carcinoma of low malignant potential of the ureter on the left side. [Fig 5]

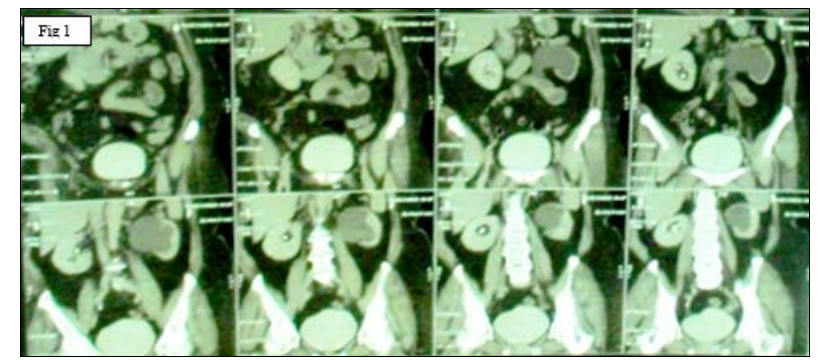

Fig 1: Computer Tomography of Kidneys, Ureters and Bladder showing left kidney measuring $12.1 \times 6.3 \mathrm{~cm}$ with moderate hydroureteronephrosis and $3 \times 1.9 \mathrm{cms}$ heterogeneously enhancing soft tissue density in the ureter.

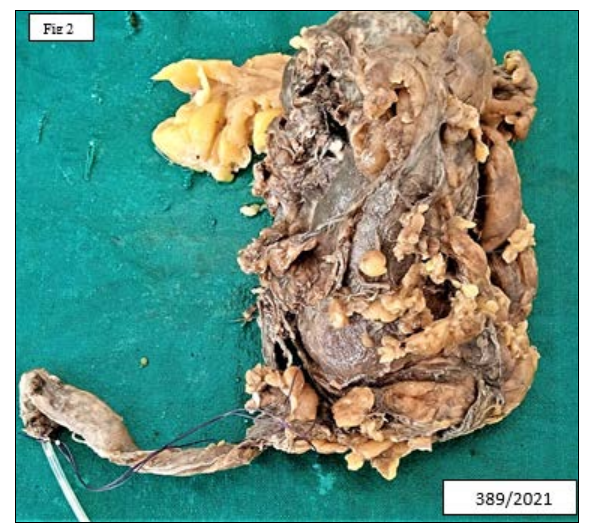

Fig 2: Macroscopic examination of left Nephroureterectomy [H.P.NO 389/2021]: Showing kidney along with perinephric fat altogether measuring $14 \times 8 \times 6 \mathrm{cms}$ with attached ureter measuring $21 \mathrm{cms}$ in length.

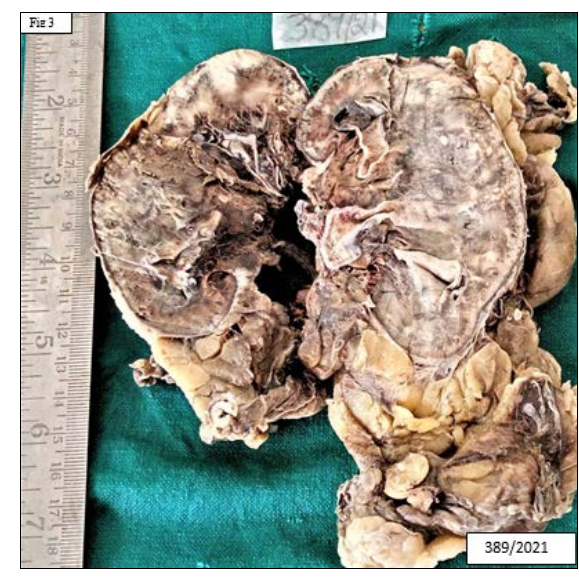

Fig 3: Macroscopic examination of left Nephroureterectomy [H.P.NO 389/2021]: Cut opened Nephroureterectomy specimen showing discretely scattered multiple greyish white nodules size ranging from $1 \mathrm{~mm}$ to $7 \mathrm{~mm}$ in diameter. Also, the renal pelvis showed dilated pelvicalyceal system.

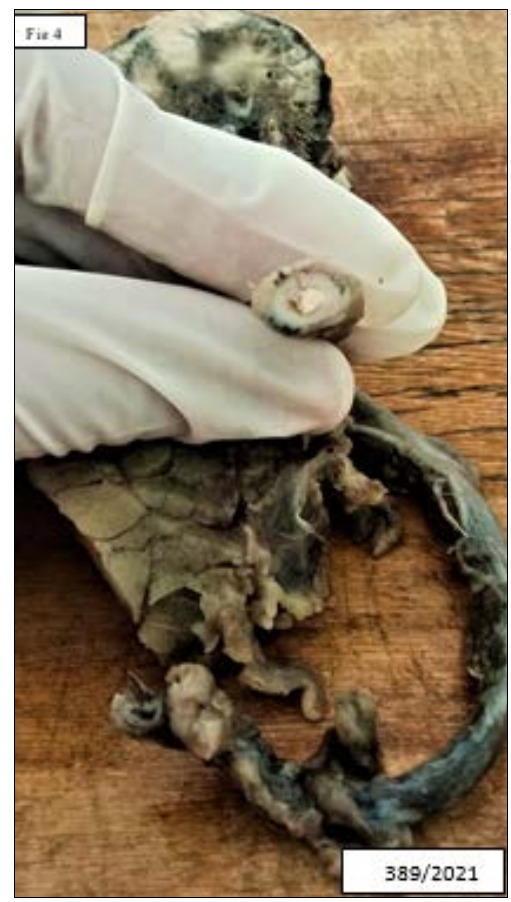

Fig 4: Macroscopic examination of left Nephroureterectomy [H.P.NO 389/2021]: Cut opened ureter showing tiny greyish white papillary growth, filling the lumen of the ureter.

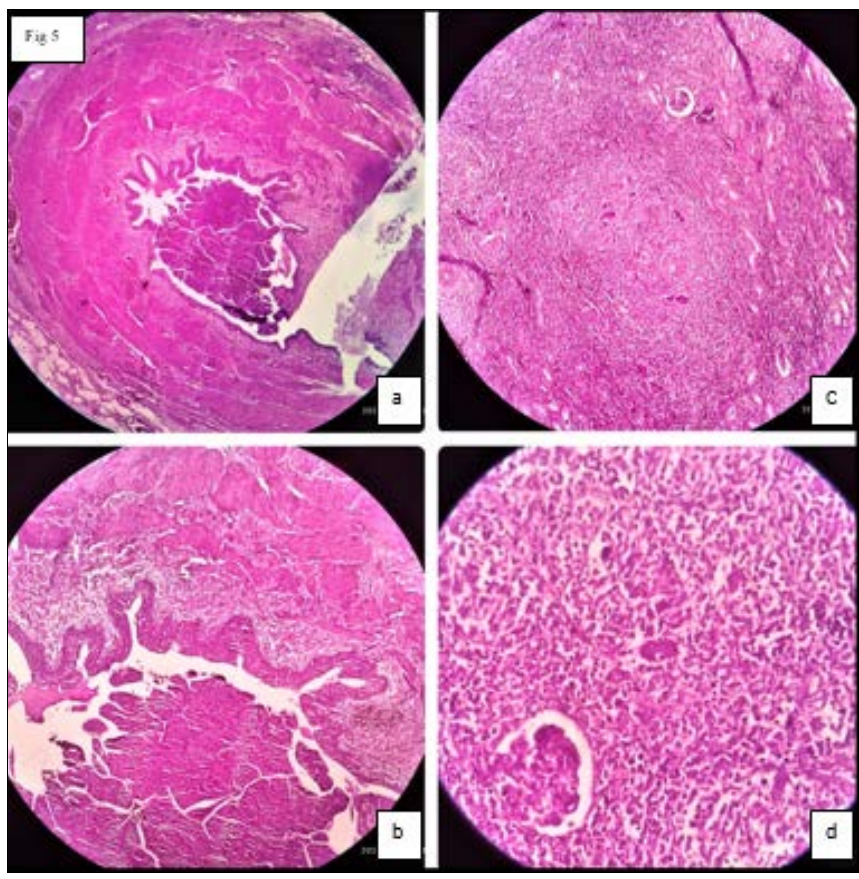

Fig 5(a-d): Photomicrograph of H\&E stained sections from Nephroureterectomy specimen [H.P.NO.389/2021]: a) \& b) scanner and low power view showing lumen of the ureter filled with papillary urothelial carcinoma. c) \& d) showing well-formed granulomas with langhans type of multinucleated giant cells seen in the renal parenchyma.

\section{Discussion}

Tuberculosis (TB), a global public health problem and is still a leading cause of death, especially in underdeveloped and developing countries ${ }^{[1]}$. According to the World Health Organization's Global TB Report 2020, globally an estimated 10.0 million people worldwide contracted tuberculosis in 2019, with an estimated 1.2 million TB fatalities among HIV-negative people and another 2 lakh deaths among HIV-positive people. Also states that India is 
one among the eight countries that accounted for two-thirds of the global TB disease burden ${ }^{[2]}$.

Any cancers emerging from urothelial cells lining the urinary tract anywhere from the renal calyces, renal pelvis, or ureter down to the ureteral orifice are referred to as UTUC. According to the American Cancer Society's Cancer Statistics, a total of 80,470 new cases of bladder cancer were diagnosed in the United States in 2019, followed by 73,820 new cases of kidney and renal pelvis malignancies, and 3,930 new cases of ureter carcinoma ${ }^{[3]}$. UTUC is still a relatively rare kind of genitourinary cancer, the majority of upper urinary tract tumors are transitional cell types, with a small number being either squamous cell type or glandular type ${ }^{[4]}$.

The co-occurrence of above mentioned two conditions both urothelial carcinoma and renal tuberculosis in the same person is uncommon, and comprehending the link between these two conditions is complex and controversial. For several years, the coexistence of tuberculosis and cancer has been a source of discussion and debate. When TB and cancer occur in the same organ system at the same time, it can be difficult to diagnose and creates a diagnostic dilemma.

The idea of an etiological link between coexisting urothelial malignancy and tuberculosis has been hypothesized by certain authors ${ }^{[5]}$. It is possible to explain the etiological relationship that the persistent inflammatory mucosal injury that sets off a series of events characterized by metaplasia and dysplasia that result in neoplastic transformation [5], as seen in some cases of colon cancer in which ulcerative lesions of intestinal tuberculosis are the precursors of colon cancers ${ }^{[6]}$. Another explanation for the association between tuberculosis and malignancy is that the cancer-associated altered host immunity can result in increased susceptibility to active tuberculous infection. It is possible to have both the above-mentioned disorders at the same location (same kidney). This is explained by the reactivation of a latent tubercular lesion or endogenous reinfection as a result of an invasion of a dormant tuberculous infection by cancer cells [7]. Another hypothesis is, the tumor peptides or antigens produced locally may potentially play a vital role that disrupts the granuloma's environment and allow the tuberculosis organisms to thrive to spread all over the tissues or organ systems.

To our knowledge, only four case reports have been published so far in English language journals to date. In 1994, Feeney et al. reported the first instance, and he estimated that the chances of these diseases occurring simultaneously in a person are about 1 in 10 billion ${ }^{[8]}$, with occurrence in the same kidney being considerably rarer.

In 2013, a national wide cohort study was done in Taiwan that found a substantial connection between urinary tuberculosis and urothelial carcinoma (1.2\% of patients with urinary TB versus $0.3 \%$ of non-urinary TB patients developed urothelial carcinoma respectively $(P<0.001)$ ). Despite this, there was no significant link was found with renal cell carcinoma $(0.2 \%$ of patients with urinary $\mathrm{TB}$ versus $0.1 \%$ of non-urinary TB patients developed renal cell carcinoma respectively $(\mathrm{P}<0.240)^{[9]}$.

In 2014, Sheray N. Chin et al. reported the third instance, in which he had reported a case of renal TB and urothelial carcinoma in the same kidney in a 72- year -old Chinese woman ${ }^{[10]}$.

In 2015, S C Hiremath et al. reported the fourth instance, in a 54-year-old man who had a history of treated urinary tuberculosis/ genitourinary Koch's ${ }^{[11]}$.

In our case report, we described a case of urothelial carcinoma and renal tuberculosis in a 54-year-old woman, as well as an attempt, has been made to know the etiological relationship between these two conditions.

After reviewing the literature, to our knowledge in our case scenario few possible explanations have been made to explain the etiological link between the concurrent occurrence of urothelial carcinoma and renal tuberculosis. The etiological link between these two conditions can be explained by the presence of long-standing ureteric calculus/urolith may be the initial event, that leads to chronic inflammatory mucosal injury causing a cascade of metaplasia and dysplasia, which leads to neoplastic transformation. This results in cancer-related changes in host immunity leading to either increased susceptibility to active tuberculosis or reactivation /endogenous reinfection of a latent tuberculous lesion by the cancer cells. Another possible explanation is, the development of carcinoma may be the primary event and the locally produced tumors peptides or antigens by the cancer cells can potentially disrupt the granuloma's environment, allowing the tuberculosis organisms to thrive to spread all over the tissues or organ systems. However in our case, it is impossible to say which condition preceded the other.

\section{Conclusion}

Renal tuberculosis and upper tract urothelial carcinoma coexisting in the same person is a relatively uncommon condition the possibility of co-occurrence should be considered, particularly in patients from TB endemic areas. Because renal tuberculosis and renal tumors have a close link, every physician, radiologist, and pathologist should be aware of this phenomenon/entity while working with patients from TB-endemic areas or those who have had a history of TB to ensure early detection and effective therapy of underlying pathology. The probable etiological factors/root causes that promote the co-occurrence of both the aforementioned conditions at the same time are also highlighted in this case report.

\section{References}

1. Blanc L, Falzon D, Fitzpatrick C, Floyd K, Garcia I, Gilpin C et al. Global Tuberculosis Control. World Health Organization: Geneva, Switzerland 2010.

2. WHO G. Global tuberculosis report 2020. Glob. Tuberc. Rep 2020.

3. Siegel RL, Miller KD, Jemal A. Cancer statistics, 2019. CA Cancer J Clin 2019;69:7-34.

4. Sternberg Stephen S, Donald Antoioli A, Stacey Mills E, Darryl Carter, Harold A. Oberman. Sternberg's Diagnostic Surgical Pathology. Volume 2. 3rd ed. Philadelphia; Wolters Kluwer Health/Lippincott Williams \& Wilkins 1999, 1879.

5. Chakravartty S, Chattopadhyay G, Ray D, Choudhury CR, Mandal S. Concomitant tuberculosis and carcinoma colon: coincidence or causal nexus?. Saudi journal of gastroenterology: official journal of the Saudi Gastroenterology Association 2010;16(4):292-294.

6. Tanaka K, Kondo S, Hattori F, Yamashita Y, Matsuda $\mathrm{M}$, Itoh $\mathrm{K}$ et al. A case of colonic carcinoma associated with intestinal tuberculosis, and an analysis of 26 cases reported in Japan. Gan no rinsho. Japan journal of 
cancer clinics 1987;33(9):1117-23.

7. Kaplan MH, Armstrong D, Rosen P. Tuberculosis complicating neoplastic disease. A review of 201 cases. Cancer 1974;33(3):850-8.

8. Feeney D, Quesada ET, Sirbasku DM, Kadmon D. Transitional cell carcinoma in a tuberculous kidney: case report and review of the literature. The Journal of urology 1994;151(4):989-91.

9. Lien YC, Wang JY, Lee MC, Shu CC, Chen HY, Hsieh $\mathrm{CH}$ et al. Urinary tuberculosis is associated with the development of urothelial carcinoma but not renal cell carcinoma: a nationwide cohort study in Taiwan. British journal of cancer 2013;109(11):2933-40.

10. Chin SN, Foster T, Char G, Garrison A. Concomitant Urothelial Cancer and Renal Tuberculosis. Case reports in urology 2014.

11. Hiremath SC, Prabha V, Hiremath MB, Ghagane SC. Research Article Genito-Urinary Tuberculosis And Urothelial Malignancy-A Case Report 2015. 